\title{
Correction to "Regularizing Effect of a Lower Order Term in Dirichlet Problems with a Singular Convection Term" [Milan J. Math. 87 (2019) 1-19]
}

Francesco Clemente

The author regrets to point out some errors in [1], even thought these do not influence the validity of the results obtained. The detailed corrections are in the following.

1. The statement of Theorem 2.3 and Remark 2.5 should be modified as follows.

Theorem 2.3. Assume (1.2)-(1.4), (1.5) with $m=1$, (1.8) and (1.10). Assume also that

$$
\lambda \geq \frac{(s+1) r}{s(r-2)} .
$$

Then there exists a weak solution $u$ of the problem (1.7) which belongs to $W_{0}^{1, q}(\Omega)$ for every $1 \leq q<q_{0}$, where $q_{0}$ is given by

$$
q_{0}:=\frac{2 \lambda s}{(\lambda+1) s+1} .
$$

Moreover $c|u|^{\lambda}$ belongs to $L^{1}(\Omega)$ and $T_{h}(u)$ belongs to $H_{0}^{1}(\Omega)$ for every $h>0$, where $T_{h}$ is the truncation at level $h$ defined in (3.32) below. Finally $u$ is the unique solution obtained by approximation in the sense of Theorem 2.2.

Remark 2.5. Theorem 2.3 improves (with respect to (1.6)) the regularity results regarding the infinite energy weak solutions when $m=1$. As a matter of fact, assumption (2.25) is equivalent to the conditions

$$
s>\frac{1}{\lambda-1} \text { and } r \geq \frac{2 \lambda s}{(\lambda-1) s-1} .
$$

A simple calculation shows that

$$
\frac{2 \lambda s}{(\lambda-1) s-1}<N
$$

The original article can be found online at https://doi.org/10.1007/s00032-019-00290-7. 
if and only if

$$
\lambda>\frac{N}{N-2} \text { and } s>\frac{N}{(N-2) \lambda-N},
$$

and

$$
\frac{2 \lambda s}{(\lambda+1) s+1}>\frac{N}{N-1}
$$

if and only if

$$
\lambda>\frac{N}{N-2} \text { and } s>\frac{N}{(N-2) \lambda-N} .
$$

Hence, it follows that we can assume (2.27) together with $r<N$ and $m=1$ in order to have $\frac{2 \lambda s}{(\lambda+1) s+1}>\frac{N}{N-1}$, provided that $\lambda>\frac{N}{N-2}$ and $s>\frac{N}{(N-2) \lambda-N}$.

2. The statement and the proof of Lemma 3.4 should be modified as follows.

Lemma 3.4. Assume (1.2)-(1.4), (1.5) with $m=1$, and (1.8)-(1.10). Then there exists a positive constant $K_{2}$ which depends only on $\alpha, r, s,\left\|c^{-s}\right\|_{L^{1}(\Omega)}$ and $\|f\|_{L^{1}(\Omega)}$, such that

$$
\int_{\left\{\left|u_{n}\right|>2 h\right\}} c(x)\left|u_{n}\right|^{\lambda} \leq \frac{K_{2}}{h^{\frac{\lambda s(r-2)}{(s+1) r}-1}}\left(\int_{\left\{\left|u_{n}\right|>h\right\}}|E(x)|^{r}\right)^{\frac{2}{r}}+\int_{\left\{\left|u_{n}\right|>h\right\}}|f(x)|
$$

for every $n \in \mathbb{N}$ and $h>0$.

Proof. We fix $h>0$ and we choose

$$
v= \begin{cases}\frac{G_{h}\left(u_{n}\right)}{\left|u_{n}\right|} & \text { if }\left|u_{n}\right|>h \\ 0 & \text { if }\left|u_{n}\right| \leq h,\end{cases}
$$

as a test function in (3.7). By (1.2), (3.3) and the fact that $|v| \leq \chi_{\left\{\left|u_{n}\right|>h\right\}}$ a.e. in $\Omega$, we have that

$$
\begin{aligned}
\alpha h \int_{\left\{\left|u_{n}\right|>h\right\}} \frac{\left|D u_{n}\right|^{2}}{\left|u_{n}\right|^{2}} & +\int_{\left\{\left|u_{n}\right|>h\right\}} c(x)\left|u_{n}\right|^{\lambda} \frac{\left|G_{h}\left(u_{n}\right)\right|}{\left|u_{n}\right|} \\
& \leq h \int_{\left\{\left|u_{n}\right|>h\right\}}|E(x)| \frac{\left|D u_{n}\right|}{\left|u_{n}\right|}+\int_{\left\{\left|u_{n}\right|>h\right\}}|f(x)|,
\end{aligned}
$$

which, using Young inequality and dropping the positive term coming from the principal part, yields to

$$
\int_{\left\{\left|u_{n}\right|>h\right\}} c(x)\left|u_{n}\right|^{\lambda} \frac{\left|G_{h}\left(u_{n}\right)\right|}{\left|u_{n}\right|} \leq \frac{h}{2 \alpha} \int_{\left\{\left|u_{n}\right|>h\right\}}|E(x)|^{2}+\int_{\left\{\left|u_{n}\right|>h\right\}}|f(x)| .
$$

We observe that $\frac{\left|G_{h}(t)\right|}{|t|}$ converges to 1 for a.e. $t \in \mathbb{R}$ when $h$ goes to 0 . Thus, letting $h \rightarrow 0$ in (3.38) and applying Fatou Lemma and dominate convergence Theorem, we obtain that

$$
\int_{\Omega} c(x)\left|u_{n}\right|^{\lambda} \leq\|f\|_{L^{1}(\Omega)}
$$


Using Hölder inequality, estimate (3.39), in particular, implies that

$$
\begin{aligned}
\left|\left\{\left|u_{n}\right|>h\right\}\right| & \leq \frac{1}{h^{\frac{s \lambda}{s+1}}} \int_{\Omega} \frac{1}{c(x)^{\frac{s}{s+1}}} c(x)^{\frac{s}{s+1}}\left|u_{n}\right|^{\frac{s \lambda}{s+1}} \\
& \leq \frac{\left\|c^{-s}\right\|_{L^{1}(\Omega)}^{\frac{1}{s+1}}\|f\|_{L^{1}(\Omega)}^{\frac{s}{s+1}}}{h^{\frac{s \lambda}{s+1}}}
\end{aligned}
$$

Then we use Hölder inequality again in (3.38) and we obtain that

$$
\begin{aligned}
& \frac{1}{2} \int_{\left\{\left|u_{n}\right|>2 h\right\}} c(x)\left|u_{n}\right|^{\lambda} \leq \int_{\left\{\left|u_{n}\right|>h\right\}} c(x)\left|u_{n}\right|^{\lambda} \frac{\left|G_{h}\left(u_{n}\right)\right|}{\left|u_{n}\right|} \\
& \quad \leq \frac{h}{2 \alpha}\left|\left\{\left|u_{n}\right|>h\right\}\right|^{\frac{r-2}{r}}\left(\int_{\left\{\left|u_{n}\right|>h\right\}}|E(x)|^{r}\right)^{\frac{2}{r}}+\int_{\left\{\left|u_{n}\right|>h\right\}}|f(x)| .
\end{aligned}
$$

Thanks to (3.40), from the previous inequality we finally deduce (3.35).

3. The proof of Theorem 2.3 should be modified as follows.

Proof of Theorem 2.3. The argument of the proof (both of existence and uniqueness) is essentially the same as the previous one. What changes is that we use Lemma 3.5 instead of Lemma 3.3 to deduce that the sequence $\left\{u_{n}\right\}$ of weak solutions of the approximate problems (3.1) constructed above is bounded in $W_{0}^{1, q}(\Omega)$ for every $1 \leq q<q_{0}$ and in $L^{\frac{\lambda s}{s+1}}(\Omega)$, with $q_{0}$ given by (2.26) above. Moreover, we use Lemma 3.4 to prove the uniform integrability of the sequence $\left\{c\left|u_{n}\right|^{\lambda-1} u_{n}\right\}$ in the following way.

We fix a measurable subset $\Omega^{\prime} \subset \Omega$ and, using Hölder inequality and Lemma 3.4, we obtain that

$$
\begin{aligned}
& \int_{\Omega^{\prime}} c(x)\left|u_{n}\right|^{\lambda}=\int_{\Omega^{\prime} \cap\left\{\left|u_{n}\right| \leq 2 h\right\}} c(x)\left|u_{n}\right|^{\lambda}+\int_{\Omega^{\prime} \cap\left\{\left|u_{n}\right|>2 h\right\}} c(x)\left|u_{n}\right|^{\lambda} \\
& \leq(2 h)^{\lambda} \int_{\Omega^{\prime}} c(x)+\frac{K_{2}}{h^{\frac{\lambda s(r-2)}{(s+1) r}-1}}\left(\int_{\left\{\left|u_{n}\right|>h\right\}}|E(x)|^{r}\right)^{\frac{2}{r}}+\int_{\left\{\left|u_{n}\right|>h\right\}}|f(x)|,
\end{aligned}
$$

for any fixed $h>0$. Thanks to (3.40) and (2.25), for every $\epsilon>0$, we can choose $h$ sufficiently large in such a way that

$$
\frac{K_{2}}{h^{\frac{\lambda s(r-2)}{(s+1) r}-1}}\left(\int_{\left\{\left|u_{n}\right|>h\right\}}|E(x)|^{r}\right)^{\frac{2}{r}}+\int_{\left\{\left|u_{n}\right|>h\right\}}|f(x)| \leq \epsilon
$$

for every $n \in \mathbb{N}$. Thus, from (4.34) we deduce that

$$
\lim _{\left|\Omega^{\prime}\right| \rightarrow 0} \int_{\Omega^{\prime}} c(x)\left|u_{n}\right|^{\lambda}=0 \quad \text { uniformly with respect to } n \text {. }
$$

Everything else in the paper remains unchanged. The author would like to apologize for the inconvenience caused. 
Publisher's Note Springer Nature remains neutral with regard to jurisdictional claims in published maps and institutional affiliations.

\section{References}

[1] F. Clemente. Regularizing effect of a lower order term in Dirichlet problems with a singular convection term. Milan J. Math. 87 (2019) 1-19, https://doi.org/10.1007/s00032-019-00290-7.

Francesco Clemente

Dipartimento di Matematica "Guido Castelnuovo"

Sapienza Università di Roma

P.le A. Moro 2

00185 Rome

Italy

e-mail: clemente@mat.uniroma1.it 